\title{
Effect of hormone replacement therapy on cardiovascular events in recently postmenopausal women: randomised trial
}

\author{
(c) $(1)$ (8)
}

\author{
Louise Lind Schierbeck registrar ${ }^{1}$, Lars Rejnmark associate professor, consultant ${ }^{2}$, Charlotte Landbo \\ Tofteng staff specialist $1^{1}$, Lis Stilgren consultant ${ }^{3}$, Pia Eiken consultant, senior endocrinologist ${ }^{4}$, \\ Leif Mosekilde professor, senior consultant ${ }^{2}$, Lars Køber professor, consultant ${ }^{5}$, Jens-Erik Beck \\ Jensen associate professor, consultant ${ }^{1}$
}

${ }^{1}$ Department of Endocrinology, Hvidovre Hospital, Kettegård alle 30, 2650 Hvidovre, Denmark; ${ }^{2}$ Department of Medicine and Department of Endocrinology and Internal Medicine, Århus University Hospital, Åarhus, Denmark; ${ }^{3}$ Department of Endocrinology, Svendborg Hospital, Svendborg, Denmark; ${ }^{4}$ Department of Cardiology, Nephrology, and Endocrinology, Hillerød Hospital, Hillerød, Denmark; ${ }^{5}$ Department of Cardiology, Rigshospitalet, Copenhagen, Denmark

\begin{abstract}
Objective To investigate the long term effect of hormone replacement therapy on cardiovascular outcomes in recently postmenopausal women. Design Open label, randomised controlled trial.

Setting Denmark, 1990-93.

Participants 1006 healthy women aged 45-58 who were recently postmenopausal or had perimenopausal symptoms in combination with recorded postmenopausal serum follicle stimulating hormone values. 502 women were randomly allocated to receive hormone replacement therapy and 504 to receive no treatment (control). Women who had undergone hysterectomy were included if they were aged $45-52$ and had recorded values for postmenopausal serum follicle stimulating hormone.

Interventions In the treatment group, women with an intact uterus were treated with triphasic estradiol and norethisterone acetate and women who had undergone hysterectomy received $2 \mathrm{mg}$ estradiol a day. Intervention was stopped after about 11 years owing to adverse reports from other trials, but participants were followed for death, cardiovascular disease, and cancer for up to 16 years. Sensitivity analyses were carried out on women who took more than $80 \%$ of the prescribed treatment for five years.
\end{abstract}

Main outcome measure The primary endpoint was a composite of death, admission to hospital for heart failure, and myocardial infarction.

Results At inclusion the women on average were aged 50 and had been postmenopausal for seven months. After 10 years of intervention, 16 women in the treatment group experienced the primary composite endpoint compared with 33 in the control group (hazard ratio $0.48,95 \%$ confidence interval 0.26 to $0.87 ; \mathrm{P}=0.015$ ) and 15 died compared with
$26(0.57,0.30$ to $1.08 ; P=0.084)$. The reduction in cardiovascular events was not associated with an increase in any cancer ( 36 in treated group $\checkmark 39$ in control group, $0.92,0.58$ to $1.45 ; \mathrm{P}=0.71$ ) or in breast cancer (10 in treated group $v 17$ in control group, $0.58,0.27$ to $1.27 ; \mathrm{P}=0.17$ ). The hazard ratio for deep vein thrombosis ( 2 in treated group $v 1$ in control group) was 2.01 ( 0.18 to 22.16 ) and for stroke (11 in treated group $v 14$ in control group) was 0.77 ( 0.35 to 1.70$)$. After 16 years the reduction in the primary composite outcome was still present and not associated with an increase in any cancer.

Conclusions After 10 years of randomised treatment, women receiving hormone replacement therapy early after menopause had a significantly reduced risk of mortality, heart failure, or myocardial infarction, without any apparent increase in risk of cancer, venous thromboembolism, or stroke.

Trial registration ClinicalTrials.gov NCT00252408.

\section{Introduction}

Hormone replacement therapy for postmenopausal women has been subject to much discussion and speculation since the 1960s. Before 2002 the effects of hormone replacement therapy were believed to be beneficial, owing to a reduction in risk of cardiovascular disease, osteoporosis, and colon cancer. ${ }^{1}$ The negative side effects-an increased risk of breast cancer and thromboembolic disease-were thought to be outweighed by the advantages, principally on the basis of results from observational studies. ${ }^{2}{ }^{3}$ In 2002 the primary results from the Women's Health Initiative showed no cardiovascular benefit from hormone replacement therapy. These conflicting results have led to the "timing hypothesis"; the idea that the differences 
in cardiovascular outcome can be accounted for by time since menopause until the start of hormone therapy. ${ }^{45}$ The observational studies mainly have shown positive cardiovascular effects, probably as a result of hormone therapy starting shortly after menopause, and the randomised studies have shown no or negative cardiovascular effects, often in women who start hormone therapy many years ( 5 to 20 ) after menopause. In meta-analyses taking age into special consideration, use of hormone therapy in younger women has been associated with a lower risk of coronary heart disease ${ }^{6}$ and reduced overall mortality. ${ }^{7}$

We used data from the Danish Osteoporosis Prevention Study (DOPS) to test whether hormone replacement therapy can reduce cardiovascular endpoints in women if started early after menopause.

\section{Methods}

The Danish Osteoporosis Prevention Study is a prospective investigator initiated multicentre trial evaluating the effect of hormone replacement therapy as primary prevention of osteoporotic fractures. The criteria for inclusion in the study were healthy, recently postmenopausal white women aged 45-58, with last menstrual bleeding 3-24 months before study entry or perimenopausal symptoms (including irregular menstruations) in combination with recorded postmenopausal serum follicle stimulating hormone values ( $>2$ standard deviations over the premenopausal mean). We included women who had had hysterectomy if they were aged 45-52 and had records showing an increase in serum follicle stimulating hormone levels. Exclusion criteria were a history of bone disease (including non-traumatic vertebral fractures on radiography), uncontrolled chronic disease, previous or current cancer or thromboembolic disease, current or past treatment with glucocorticoids for more than six months, current or previous use of hormone replacement therapy within the past three months, and alcohol or drug dependency.

Between 1990 and 1993, 2016 women were enrolled in a prospectively followed cohort. Of these, 1006 were randomly allocated (open label) to receive hormone replacement therapy $(n=502)$ or no treatment $(n=504)$; the remaining 1010 women had a personal choice (of these, 221 opted for hormone replacement therapy). The results presented here are based solely on the randomised groups. Recruitment has been described in detail elsewhere. ${ }^{8}$ Participants were recruited by direct mailing to a random sample $(n=47720)$ of women from the general background population. ${ }^{9}$ In the invitation letter women were asked to participate in a study on prevention of postmenopausal osteoporosis. Participants were stratified according to centre and randomly allocated to either hormone replacement therapy or no treatment in blocks of 10 , using sealed envelopes.

Participants gave informed consent before the study.

The women in the treated group with an intact uterus started treatment with $2 \mathrm{mg}$ synthetic $17-\beta$-estradiol for 12 days, $2 \mathrm{mg}$ $17-\beta$-estradiol plus $1 \mathrm{mg}$ norethisterone acetate for 10 days, and $1 \mathrm{mg}$ 17- $\beta$-estradiol for six days (Trisekvens; Novo Nordisk, Denmark). In women who had undergone hysterectomy, first line treatment was $2 \mathrm{mg}$ 17- $\beta$-estradiol a day (Estrofem; Novo Nordisk, Denmark). Other treatment modalities were offered to those who experienced side effects or insufficient relief of symptoms. ${ }^{10}$

All participants underwent a physical examination and biochemical screening at baseline. They were subsequently seen after six months, one year, and two, three, five, and 10 years. The study drugs were posted to the women randomised to hormone replacement therapy, and they were offered an annual visit. We advised the women that if they had health concerns they should contact their own general practitioner or gynaecologist. The planned duration of the study was 20 years. However, as data published from other trials at the time of the 10 year visit indicated that use of hormone replacement therapy might result in more harm than benefit in postmenopausal women we advised our study participants to stop treatment. ${ }^{11}$ After their 10 year visit we followed the participants in national registers, which provide data on all hospital contacts or deaths.

\section{Assessment of mortality and admission to hospital due to cardiovascular events or cancer}

The primary endpoint for this study was a composite of death, admission to hospital for myocardial infarction, or heart failure. We prespecified and adjudicated cardiovascular disease as well as cancer as safety outcome measures. Secondary endpoints were the individual components of the primary endpoint and admission to hospital for stroke. Safety endpoints included death or a diagnosis of breast cancer or other cancer grouped together, and admission to hospital for pulmonary embolism or deep venous thrombosis. Evaluations of endpoints were carried out with a PROBE (Prospectively, Randomised, Open with Blinded Endpoint evaluation) design.

On 16 June 2008 we ended our follow-up period by retrieving data on all participants from the Danish civil registration system and the national hospital discharge register. In the Danish civil registration system we identified all women who had died or emigrated during follow-up, as this register has electronic records on all changes in vital status, including change of address and date of death for the entire Danish population since 1968.

Using the Danish national hospital discharge register, which covers all contacts to Danish hospitals, we identified women who had been admitted to hospital for a cardiovascular event. The register was founded in 1977 and includes information on discharge diagnoses and date of discharge assigned exclusively by the doctor at discharge according to the International Classification of Diseases, eighth revision until the end of 1993 and the 10th revision from 1994. The register has nationwide coverage of hospitals with an almost $100 \%$ completeness of recordings and a high precision of diagnoses. ${ }^{12}$ Using this register, we identified all study participants who had been assigned a diagnosis of cardiovascular disease classified as myocardial infarction (ICD-10 code I21), heart failure (ICD-10 code I42 and I50), and stroke (ICD-10 code I60 to I69, which covers ischaemic as well as non-ischaemic stroke). For the composite endpoint we used the date of the first incident.

Total cancer included all cancer diagnoses (ICD-10 codes C01 to C99) except non-melanoma skin cancer (ICD-10 codes C44). We defined other cancer as total cancer except for breast cancer (ICD-10 code C50), which was independently surveyed and monitored. Breast cancer was analysed both as a composite endpoint (with mortality) and as breast cancer only. For composite endpoints, we used the date of first incident. Pulmonary embolism (ICD-10 code I26.9) and deep vein thrombosis (ICD-10code I80.1 to 80.3) were registered separately.

\section{Statistical analysis}

Only the randomised participants are considered in this study $(n=1006)$, and all analyses are done on the intention to treat population, except when mentioned specifically in sensitivity 
analyses. The analyses were carried out, with 1 august 2002 as the stopping date, about 10 years after randomization (when the randomised treatment was stopped). So as not to miss major long term effect, we carried out secondary analyses with an additional six years of non-randomised follow-up. Because women who had undergone hysterectomy in the treatment group received oestrogen only, post-hoc analyses were carried out for this group; a total of 192 women had undergone hysterectomy, of whom 95 were randomised to treatment. As only three women had undergone complete oophorectomy, no subgroup analyses were done. Unless otherwise stated, baseline data are expressed as means (standard deviations). We tested dichotomous variables with a $\chi^{2}$ test and continuous variables with students $t$ test. All tests were two sided, and we considered $\mathrm{P}<0.05$ to be statistically significant. The survival data and the composite endpoint are presented using the Kaplan-Meier method and analysed by a $\log$ rank test. We carried out analyses as time to first event, thus we counted the women only once at their first endpoint of that analysis. Hazard ratios (95\% confidence intervals) were determined using Cox proportional hazards regression analyses. We repeated the Cox regression analyses adjusted for age. Age was included owing to a difference of 0.5 years between the two randomised groups. With a hazard ratio for the primary endpoint of less than 0.7 the study had a power of at least $80 \%$ to detect a difference between the two groups. We tested model assumptions including linearity of continuous variables, the proportional hazards assumption, and absence of interactions and found them to be valid unless otherwise indicated. We carried out sensitivity analyses on women who took more than $80 \%$ of the prescribed treatment for five years. Statistical analyses were done with SAS version 9.2.

\section{Results}

Of 1006 recently postmenopausal women or women with perimenopausal symptoms and recorded postmenopausal serum follicle stimulating hormone levels included in the study, 502 were randomly allocated to hormone replacement therapy and 504 to no treatment fig $1 \Downarrow$ ). The women had a mean age of 49.7 ( 2.8 years), mean body mass index of 25.2 (4.4), and mean time since menopause of 0.59 ( 0.64$)$ years (about seven months).

Their mean blood pressure was $130 / 81 \mathrm{~mm} \mathrm{Hg}$ and $43 \%$ of the women were smokers at the time of inclusion. Women in the control group were 0.47 years (about 5.7 months) older $(\mathrm{P}=0.006)$ than those in the treated group, whereas other variables did not differ significantly between the groups (table $1 \Downarrow)$. Only $22(2 \%)$ of the women had used hormone replacement therapy previously, for a median duration of 1 year (interquartile range $0-5$ years).

\section{Clinical outcome}

After a mean duration of 10.1 years of randomised treatment the women were encouraged to discontinue use of hormone therapy on 1 August 2002 following adverse outcomes in the Women's Health Initiative and hence the first analyses were done with 1 August 2002 as the stop date. After termination of randomisation, the women were followed for an additional 5.7 years for a total mean follow-up time of 15.8 years. No participants were lost to follow-up, but two women were censored at time of emigration (one in each randomisation group). At five years, $75 \%$ of the women adhered to the randomisation arm to which they were allocated for $80 \%$ or more of the time. Results on osteoporosis in the Danish Osteoporosis Prevention Study have been published previously. ${ }^{913}$

\section{Data for 10 years of randomised treatment}

The primary endpoint occurred in 49 women (33 in control group $v 16$ in treated group; hazard ratio $0.48,95 \%$ confidence interval 0.26 to $0.87 ; \mathrm{P}=0.015)$ and $0.49(0.27$ to $0.89 ; \mathrm{P}=0.019)$ when adjusted for age (fig $2 \Downarrow$ ). During the intervention period 41 women died (26 in control group $v 15$ in treated group; 0.57 , 0.30 to $1.08 ; \mathrm{P}=0.084)$. Heart failure was diagnosed in eight participants ( 7 in control group $v 1$ in treated group; 0.14, 0.02 to $1.16 ; \mathrm{P}=0.07)$ and myocardial infarction was diagnosed in five participants ( 4 in control group $v 1$ in treated group; 0.25 , 0.03 to $2.21 ; \mathrm{P}=0.21$ ).

Stroke rates did not differ between the groups (14 in control group $v 11$ in treated group; $0.77,0.35$ to $1.70 ; \mathrm{P}=0.70)$. The rate of venous thromboembolism was low and did not differ significantly between groups. Three women had confirmed deep vein thrombosis ( 1 in control group $v 2$ in treated group; 2.01, 0.18 to 22.16 ) and only one woman (control group) was admitted to hospital with pulmonary embolism.

The occurrence of any cancer did not differ significantly (39 in control group $v 36$ in treated group; $0.92,0.58$ to $1.45 ; \mathrm{P}=0.71$ ) or breast cancer (17 in control group $v 10$ in treated group, 0.58 , 0.27 to 1.27 ; $\mathrm{P}=0.17$; fig 4 ). The occurrence of other cancers did not differ significantly (25 in control group $v 26$ in treated group; $1.04,0.60$ to $1.80 ; \mathrm{P}=0.88$ ): three women in the control group had a diagnosis of both breast cancer and other cancer. The composite endpoint mortality or breast cancer applied to 40 women in the control group and 22 in the treated group $(0.54$, 0.32 to $0.91, \mathrm{P}=0.020$ ).

Fig $3 \Downarrow$ shows the subgroup analyses of the different endpoints. When the groups were divided according to age (more than or less than median age 50 years) the hazards ratios for the primary endpoints were 0.63 ( 0.29 to 1.36 ) for women aged more than 50 and 0.35 ( 0.13 to 0.89$)$ for those aged less than 50 . The hazard ratios for the combined endpoint mortality and breast cancer in these age groups were 0.36 ( 0.17 to 0.79 ) and 0.77 ( 0.38 to 1.57 ), respectively. Fig $4 \Downarrow$ shows the subgroup analyses of the safety endpoints.

Deaths due to cardiovascular causes occurred in 18 women in the control group and five in the treated group. Deaths due to non-cardiovascular causes occurred in eight women in the control group and 10 in the treated group.

\section{Data from 16 years of total follow-up}

The composite primary trial endpoint of death or myocardial infarction or heart failure occurred in 86 women (53 in control group $v 33$ in treated group; $0.61,0.39$ to $0.94 ; \mathrm{P}=0.02$, fig $5 \Downarrow)$. Adjustment for age did not change the results $(0.62,0.40$ to 0.96). The Kaplan-Meier curves indicate that soon after randomisation the difference between treatment groups began to diverge and there was no apparent change after year 10 when women were advised to stop hormone therapy owing to adverse reports from other trials. During the 16 years 67 women died (40 in control group $v 27$ in treated group; 0.66, 0.41 to 1.08; $\mathrm{P}=0.10$; fig 5). Heart failure was diagnosed in 11 participants (8 in control group $v 3$ in treated group; $0.37,0.10$ to 1.41 ; $\mathrm{P}=0.15)$ and myocardial infarction was diagnosed in 16 participants (11 in control group $v 5$ in treated group; 0.45, 0.16 to $1.31 ; \mathrm{P}=0.14)$.

Stroke rates did not differ between groups, with 21 cases in the control group and 19 in the treated group $(0.89,0.48$ to 1.65 ; $\mathrm{P}=0.71$ ). The rate of venous thromboembolism and pulmonary embolism was low and there was no significant difference between groups (fig $6 \Downarrow$ ). Nine women had confirmed deep vein thrombosis ( 5 in control group $v 4$ in treated group; $0.80,0.22$ 
to $2.99 ; \mathrm{P}=0.74$ ), and only four women were admitted to hospital with pulmonary embolism ( 3 in control group $v 1$ in treated group; $0.33,0.04$ to $3.21 ; \mathrm{P}=0.34)$.

The groups did not differ significantly for breast cancer (26 in control group $v 24$ in treated group; $0.90,0.52$ to $1.57 ; \mathrm{P}=0.72$ ) or for other cancers (43 in control group $v 52$ in treated group; $1.21,0.81$ to $1.82 ; \mathrm{P}=0.35$, fig 6 ). A significant interaction was found between hormone replacement therapy and age at baseline for the composite endpoint mortality or breast cancer $(\mathrm{P}=0.028)$ with the younger women $(<50$ years) receiving hormone therapy having a significantly reduced risk $(0.49,0.28$ to $0.87, \mathrm{P}=0.015$, fig 6). Women who had undergone hysterectomy $(\mathrm{n}=192)$ and received oestrogen alone had a decreased risk of death or breast cancer compared with women in the control group $(0.42,0.18$ to $0.97 ; \mathrm{P}=0.043$; fig 6 ). Owing to a limited number of women who had undergone hysterectomy, data did not have the power to differentiate between women randomised to oestrogen only versus control compared with women randomised to combination hormone therapy (17- $\beta$-estradiol and norethisterone acetate) in relation to the composite endpoint (death, myocardial infarction, or heart failure), but the results were similar to those from combined therapy $(0.52,0.21$ to $1.30, \mathrm{P}=0.16)$.

In the control group 23 deaths were due to cardiovascular causes and 17 to non-cardiovascular causes. In the treatment group six deaths were due to cardiovascular causes and 21 to noncardiovascular causes

In the early 1990s a large proportion of the female Danish population were smokers, but the hazard ratios were similar between smokers and non-smokers in this study and there was no interaction between smoking and treatment for the primary endpoint or for mortality alone (data not shown).

Sensitivity analyses with women taking more than $80 \%$ of the prescribed medication after five years supported the data for all endpoints in the randomisation phase as well as the total follow-up (data not shown).

\section{Discussion}

In this randomised trial including 1006 women we found a significantly decreased risk of the composite endpoint of death, heart failure, or myocardial infarction when hormone replacement therapy was started early in postmenopause. The beneficial effect occurred in the 10 years randomisation phase and was maintained for an additional six years of

non-randomised follow-up. The trend for all components of the endpoint was in the same direction (figs 3 to 6 ) and this finding was not associated with an increased risk of cancer, stroke, deep vein thrombosis, or pulmonary embolism. Thus this study implies that hormone therapy started in recently menopausal women and continued for a prolonged duration does not increase or provoke adverse cardiovascular events such as mortality, stroke, heart failure, or myocardial infarction. The rate of breast cancer and other cancer was not increased in the present study, but because of the potential time lag a longer follow-up may be necessary to make more definite conclusions. Secondly, healthier women may not develop adverse events quickly. Moreover, the number of events was low, adding uncertainty to the results. However, the beneficial effect is supported by the observation that the compliant participants did not have an increased risk of adverse events. Observational studies ${ }^{23}$ suggested that hormone replacement therapy could reduce cardiovascular events, but results have been explained by heavy confounding owing to favourable differences in a priori risks between women choosing and not choosing hormone therapy.
When the first results from the Women's Health Initiative were reported in 2002, the Danish Osteoporosis Prevention Study intervention (randomisation phase) was stopped because of a reported excess risk of breast cancer and adverse cardiovascular events. ${ }^{11}$ The discrepancy between that trial and the Danish Osteoporosis Prevention Study may be explained by a difference in medication or in the characteristics of women included in the trials. In the present study we used synthetic $17-\beta$-estradiol, whereas conjugated equine oestrogen was administered in the Women's Health Initiative. The gestogens were norethisterone acetate and medroxyprogesterone, in the present study and Women's Health Initiative, respectively. The mean age at randomisation in the Danish Osteoporosis Prevention Study was much younger than in the Women's Health Initiative (50 years $v 64$ years) and the average time from menopause when the women were randomised was considerably shorter $(0.7 v$ 10 years). Thus at randomisation in the Women's Health Initiative most of the women had started menopause many years earlier ${ }^{11}$ whereas $98 \%$ of the participants in the present study had not taken hormone replacement therapy by study start.

Our results substantiate a later subgroup analysis from the Women's Health Initiative of women aged less than 60 and postmenopausal for less than 10 years when randomised, where a non-significant reduction in coronary heart disease and mortality was reported..$^{14}$ In the Women's Health Initiative, women receiving conjugated equine oestrogen alone, aged 50 to 59, experienced a reduced risk of the combined endpoint myocardial infarction, coronary death, coronary artery bypass grafting, or percutaneous coronary intervention, and a non-significantly reduced risk of myocardial infarction or coronary death. ${ }^{15}$ Our trial results support the timing hypothesis ${ }^{4-7}$ and a series of meta-analyses of randomised controlled trials that indicate a significant reduction in coronary heart disease and mortality in women who were randomised before age 60 or within 10 years of menopause. ${ }^{67}$ A bayesian meta-analysis on randomised controlled trials including women of mean age 55 supported a positive effect of hormone replacement therapy on mortality (relative risk $0.73,95 \%$ confidence interval 0.52 to 0.96$).{ }^{16}$ However, not all reports support the timing hypothesis. Combining the results from the observational study of conjugated equine oestrogen and medroxyprogesterone and randomised trial data from the Women's Health Initiative to differentiate between women starting hormone therapy within five years of menopause or later, the risk of coronary heart disease was non-significantly increased, and an additional non-significant difference owing to timing of starting hormone therapy. ${ }^{17}$

\section{Thromboembolism}

The of risk stroke, deep vein thrombosis, and pulmonary embolism was not increased in women in the present study, although a small number of deep vein thrombosis and pulmonary embolism events occurred, which makes interpretations uncertain. However these were in young, healthy women, who were not expected to develop deep vein thrombosis or pulmonary embolism. These results are incongruous with most other studies, observational ${ }^{2}{ }^{3}$ as well as randomised. ${ }^{18-20}$ This may be due to the differences in the administered hormones; $17-\beta$-estradiol has been reported to be less thrombogenic than conjugated equine oestrogen. ${ }^{21}{ }^{22}$ In human aortic endothelial cells $17-\beta$-estradiol is superior to conjugated equine oestrogen in increasing the production of nitric oxide, partially because of a higher ability to activate the production of endothelial nitric oxide synthase..$^{23}$ However, the results in humans have been more ambiguous. One study in 88 postmenopausal women found 
no difference between treatment regimens and haemostatic variables,${ }^{24}$ and in healthy postmenopausal women combined $17-\beta$-estradiol and norethisterone acetate has resulted in reduced levels of fibrinogen, factor VIIc, antithrombin, tissue plasminogen activator antigen, and plasminogen activator inhibitor-1 antigen and increased levels of fibrin/fibrinogen degradation products (D-dimer). ${ }^{25}$

\section{Mechanisms}

Several other mechanisms may explain the beneficial effects of hormone replacement therapy on cardiovascular endpoints. Different hormone replacement therapy regimens have been found to have positive effects on lipid metabolism ${ }^{26-28}$ and the combination 17- $\beta$-estradiol with norethisterone acetate has been shown to lower total cholesterol levels and improve endothelial function in healthy postmenopausal women. ${ }^{29}$ In other studies, however, gestogens have been found to blunt the positive effects of oestrogen. ${ }^{26}{ }^{30}$ The type of progestogen used may be important, as natural progesterone seems to have more beneficial effects on the cardiovascular system than does

medroxyprogesterone acetate. ${ }^{30}$ Moreover, body composition is favourably affected by hormone replacement therapy, where a significant increase in fat mass and trunk fat has been observed in control treated compared with hormone replacement therapy treated women after five years. ${ }^{32}$

The difference in cholesterol levels between groups (lower levels in hormone therapy group) seen after five years in the present study (data not shown) is in line with several previous studies ${ }^{26-28}$ and may be one of the factors associated with the decreased risk in cardiovascular events. Also, healthy postmenopausal women receiving unopposed $17-\beta$-estradiol had significantly less progression of carotid intima media thickness compared with those receiving placebo. ${ }^{27}$ However, this cannot be reproduced for women with established coronary artery atherosclerosis. ${ }^{33}$ The clinical effect may be even greater for the combination treatment $17-\beta$-estradiol and norethisterone acetate. In rabbits this combination resulted in an additional preventive atherogenic effect compared with 17- $\beta$-estradiol alone or with placebo-with higher doses of norethisterone acetate resulting in lower aortic cholesterol content. This was only partly explained by the lowering effect on serum lipid and lipoprotein levels. ${ }^{34}$

\section{Unopposed oestrogen and breast cancer}

For the subgroup of women who had undergone hysterectomy and who received unopposed $17-\beta$-estradiol/control we found a significant reduction in the combined endpoint of mortality or breast cancer in the treatment group. This is in accordance with findings from the Women's Health Initiative, where 10 739 women who had undergone hysterectomy were randomised to conjugated equine oestrogen or placebo $0.625 \mathrm{mg} / \mathrm{day}$; all women (irrespective of age) in the conjugated equine oestrogen arm experienced a reduced risk of breast cancer (hazard ratio $0.77,95 \%$ confidence interval 0.62 to 0.95$).{ }^{35}$ Furthermore, in the present study, there seemed to be a non-significant increase in breast cancer in the treatment arm after discontinuation of treatment (hazard ratio 0.58 after 10 years and 0.90 after 16 years) predominantly in the women aged more than 50, although none of these differences were statistically significant.

Previously, starting hormone replacement therapy early has been considered unfavourable for breast cancer, ${ }^{36}{ }^{37}$ and conclusions cannot be drawn from the presented data. A recent trial, the Kronos Early Estrogen Prevention Study, ${ }^{38}$ may reveal more on whether timing, type of oestrogen, and route of being administered can account for the differences across previous studies.
We found a borderline interaction with age and breast cancer suggesting that hormone replacement therapy reduces the risk of breast cancer in women aged less than 50 (or at least it was not harmful), but data were not consistent over time. We find it difficult to draw firm conclusions from these data.

\section{Strengths and limitations of the study}

The present study was randomised but an open label trial with no placebo or blinding, and endpoints were determined without knowledge of treatment allocation, using a PROBE design. Despite the use of such a design, when endpoints were evaluated we cannot exclude that some diagnoses were more often suspected by doctors who knew which drug the women were taking. The randomised part of the present study only comprised 1006 women, but significant findings in this size is more likely to be of clinical relevance. Another weakness is that not all women adhered to treatment group or first-line treatment (although a high proportion did) and the results from the intention to treat analysis might be slightly different if adherence had been better, not displaying the full magnitude of the effect. However, this is not different from randomised studies in general. Finally, osteoporosis was an endpoint in the original design of the study; nevertheless, important information on participants for cardiovascular endpoints was assessed at baseline, and cardiovascular and cancer endpoints were prespecified as important safety endpoints.

Randomisation is an important variable in clinical trials and few hormone replacement therapy trials have randomised healthy postmenopausal women. With the longest duration of randomised treatment and complete and long-term follow up, the present study provides a unique opportunity to study the clinical implications of long term hormone therapy started in young postmenopausal women within three to 24 months of menopause when randomised. The additional six years of follow-up after discontinuation of the randomised treatment is difficult to interpret, but the present data are reassuring as we found no apparent increase in cancer or cardiovascular events. Secondly, it is uncertain whether women continued treatment after information of the results of the Women's Health Initiative in 2002.

Participants in randomised trials generally are healthier than the background population, therefore extrapolation to effect in other groups is difficult. However, this is no different from randomised trials in general. Using a population based approach, recruiting participants by direct mail to a random sample of Danish women in the perimenopausal to early postmenopausal age range, we believe that our study participants were as representative as possible for a randomised trial. Moreover, use of an open trial design has been shown to enhance participant recruitment and retention, which may improve generalisability. ${ }^{39}$

An inclusion criterion to the present study was proximity to menopause and hence the design resembles a realistic clinical situation of the timing where women are most likely to start using hormone replacement therapy. The Danish civil registration number register enabled us to retrieve hospital diagnoses and dates of death on all included women, and only the two (one participant in each group) that emigrated were lost to follow-up.

\section{Conclusions}

This is the first randomised trial to study healthy women treated early in postmenopause with $17-\beta$-estradiol and norethisterone acetate, and the only study with a 10 year randomised intervention. Additionally the women were followed for a further 
six years after discontinuation of randomised treatment. Our findings suggest that initiation of hormone replacement therapy in women early after menopause significantly reduces the risk of the combined endpoint of mortality, myocardial infarction, or heart failure. Importantly, early initiation and prolonged hormone replacement therapy did not result in an increased risk of breast cancer or stroke.

Novo Nordisk, Novartis, and Leo Pharma Denmark provided the study drug free of charge.

Contributors: LM contributed to the original design. J-EBJ, LLS, and LK planned the present study. LM, PE, LLS, CLT, LR, and J-EBJ implemented the study at different sites. LR retrieved data from national registers. LR, LK, CLT, and LLS organised the database. LLS and LK had principal responsibility for the statistical analysis of the data and are the guarantors. LLS, LK, LR, and J-EBJ were active in the interpretation of the results. The manuscript was drafted by LLS and reviewed by all authors. All authors have approved the final report. Funding: This study was funded by the University of Aarhus, Karen Elise Jensen's Foundation, Novo Nordic, Novartis, and LEO Pharma. None of the funders had any influence on the study design, interpretation of data, or the decision to publish the results.

Competing interests: All authors have completed the ICMJE uniform disclosure form at www.icmje.org/coi_disclosure.pdf (available on request from the corresponding author) and declare: $\mathrm{PE}$ is on the advisory board of Nycomed, Novartis and is involved in the speaker's bureau with Amgen, Novartis, Eli Lilly, and GSK; J-EBJ is on the advisory board and speaker's bureau of Nycomed, Eli Lilly, Novartis, MSD, and Amgen; no financial relationships with any organisations that might have an interest in the submitted work in the previous three years; and no other relationships or activities that could appear to have influenced the submitted work.

Ethical approval: This study was approved by the Danish National Committee on Health Research Ethics in 1990.

Data sharing: Additional data from the study database are available on request from the corresponding author louise.schierbeck@gmail.com.

1 Grodstein F, Stampfer M. The epidemiology of coronary heart disease and estrogen replacement in postmenopausal women. Prog Cardiovasc Dis 1995;38:199-210.

2 Grodstein F, Stampfer MJ. Estrogen for women at varying risk of coronary disease. Maturitas 1998:30:19-26.

3 Grodstein F, Stampfer MJ, Manson JE, Colditz GA, Willett WC, Rosner B, et al. Postmenopausal estrogen and progestin use and the risk of cardiovascular disease. $N$ Engl J Med 1996;335:453-61.

4 Dubey RK, Imthurn B, Barton M, Jackson EK. Vascular consequences of menopause and hormone therapy: importance of timing of treatment and type of estrogen. Cardiovasc Res 2005:66:295-306.

5 Hodis HN, Mack WJ. A "window of opportunity:" the reduction of coronary heart disease and total mortality with menopausal therapies is age- and time-dependent. Brain Res 2011;1379:244-52.

6 Salpeter SR, Walsh JM, Greyber E, Salpeter EE. Brief report: coronary heart disease events associated with hormone therapy in younger and older women. A meta-analysis. $J$ Gen Intern Med 2006;21:363-6.

7 Salpeter SR, Walsh JM, Greyber E, Ormiston TM, Salpeter EE. Mortality associated with hormone replacement therapy in younger and older women: a meta-analysis. $J$ Gen Intern Med 2004;19:791-804

8 Mosekilde L, Hermann AP, Beck-Nielsen H, Charles P, Nielsen SP, Sorensen OH. The Danish Osteoporosis Prevention Study (DOPS): project design and inclusion of 2000 normal perimenopausal women. Maturitas 1999;31:207-19.

9 Mosekilde L, Beck-Nielsen H, Sorensen OH, Nielsen SP, Charles P, Vestergaard P, et al. Hormonal replacement therapy reduces forearm fracture incidence in recent postmenopausal women-results of the Danish Osteoporosis Prevention Study. Maturitas 2000;36:181-93.

10 Vestergaard P, Hermann AP, Gram J, Jensen LB, Kolthoff N, Abrahamsen B, et al. Improving compliance with hormonal replacement therapy in primary osteoporosis prevention. Maturitas 1997;28:137-45.

11 Rossouw JE, Anderson GL, Prentice RL, LaCroix AZ, Kooperberg C, Stefanick ML, et al. Risks and benefits of estrogen plus progestin in healthy postmenopausal women: principal results from the Women's Health Initiative randomized controlled trial. JAMA 2002;288:321-33.

12 Andersen TF, Madsen M, Jorgensen J, Mellemkjoer L, Olsen JH. The Danish National Hospital Register. A valuable source of data for modern health sciences. Dan Med Bull 1999;46:263-8.

13 Jensen LB, Vestergaard P, Hermann AP, Gram J, Eiken P, Abrahamsen B, et al. Hormone replacement therapy dissociates fat mass and bone mass, and tends to reduce weight gain in early postmenopausal women: a randomized controlled 5 -year clinical trial of the Danish Osteoporosis Prevention Study. J Bone Miner Res 2003:18:333-42.

14 Rossouw JE, Prentice RL, Manson JE, Wu L, Barad D, Barnabei VM, et al. Postmenopausal hormone therapy and risk of cardiovascular disease by age and years since menopause. JAMA 2007;297:1465-77.

15 Hsia J, Langer RD, Manson JE, Kuller L, Johnson KC, Hendrix SL, et al. Conjugated equine estrogens and coronary heart disease: the Women's Health Initiative. Arch Intern Med 2006;166:357-65.

16 Salpeter SR, Cheng J, Thabane L, Buckley NS, Salpeter EE. Bayesian meta-analysis of hormone therapy and mortality in younger postmenopausal women. Am J Med 2009;122:1016-22.

17 Prentice RL, Manson JE, Langer RD, Anderson GL, Pettinger M, Jackson RD, et al. Benefits and risks of postmenopausal hormone therapy when it is initiated soon after menopause. Am J Epidemiol 2009;170:12-23.

18 Gabriel SR, Carmona L, Roque M, Sanchez GL, Bonfill X. Hormone replacement therapy for preventing cardiovascular disease in post-menopausal women. Cochrane Database Syst Rev 2005;(2):CD002229.

19 Sare GM, Gray LJ, Bath PM. Association between hormone replacement therapy and subsequent arterial and venous vascular events: a meta-analysis. Eur Heart $J$ 2008;29:2031-41.

20 Nelson HD, Humphrey LL, Nygren P, Teutsch SM, Allan JD. Postmenopausal hormone replacement therapy: scientific review. JAMA 2002;288:872-81.

21 Leng XH, Zhang W, Nieswandt B, Bray PF. Effects of estrogen replacement therapies on mouse platelet function and glycoprotein VI levels. Circ Res 2005;97:415-7.

22 Jayachandran M, Mukherjee R, Steinkamp T, LaBreche P, Bracamonte MP, Okano H, et al. Differential effects of 17beta-estradiol, conjugated equine estrogen, and raloxifene on mRNA expression, aggregation, and secretion in platelets. Am J Physiol Heart Circ Physiol 2005;288:H2355-62.

23 Novensa L, Selent J, Pastor M, Sandberg K, Heras M, Dantas AP. Equine estrogens impair nitric oxide production and endothelial nitric oxide synthase transcription in human endothelial cells compared with the natural 17 \{beta\}-estradiol. Hypertension 2010;56:405-11.

24 Taner MZ, Ozpolat E, Taskiran C, Onan MA, Gursel T, Karabulut E, et al. Effects of four different regimens of hormone replacement therapy on hemostatic parameters: a prospective randomized study. Maturitas 2006;53:267-73.

25 Norris LA, Joyce M, O'Keeffe N, Sheppard BL, Bonnar J. Haemostatic risk factors in healthy postmenopausal women taking hormone replacement therapy. Maturitas 2002;43:125-33.

26 Effects of estrogen or estrogen/progestin regimens on heart disease risk factors in postmenopausal women. The Postmenopausal Estrogen/Progestin Interventions (PEPI) Trial. The Writing Group for the PEPI Trial. JAMA 1995;273:199-208.

27 Hodis HN, Mack WJ, Lobo RA, Shoupe D, Sevanian A, Mahrer PR, et al. Estrogen in the prevention of atherosclerosis. A randomized, double-blind, placebo-controlled trial. Ann Intern Med 2001;135:939-53.

28 Hulley S, Grady D, Bush T, Furberg C, Herrington D, Riggs B, et al. Randomized trial of estrogen plus progestin for secondary prevention of coronary heart disease in postmenopausal women. Heart and Estrogen/progestin Replacement Study (HERS) Research Group. JAMA 1998;280:605-13.

29 Guzic-Salobir B, Keber I, Seljeflot I, Arnesen H, Vrabic L. Combined hormone replacement therapy improves endothelial function in healthy postmenopausal women. $J$ Intern Med 2001;250:508-15.

30 Simoncini T, Mannella P, Fornari L, Caruso A, Varone G, Genazzani AR. In vitro effects of progesterone and progestins on vascular cells. Steroids 2003;68(10-13):831-6.

31 Rosano GM, Webb CM, Chierchia S, Morgani GL, Gabraele M, Sarrel PM, et al. Natural progesterone, but not medroxyprogesterone acetate, enhances the beneficial effect of estrogen on exercise-induced myocardial ischemia in postmenopausal women. J Am Coll Cardiol 2000;36:2154-9.

32 Kristensen K, Pedersen SB, Vestergaard P, Mosekilde L, Richelsen B. Hormone replacement therapy affects body composition and leptin differently in obese and non-obese postmenopausal women. J Endocrinol 1999;163:55-62.

33 Hodis HN, Mack WJ, Azen SP, Lobo RA, Shoupe D, Mahrer PR, et al. Hormone therapy and the progression of coronary-artery atherosclerosis in postmenopausal women. $\mathrm{N}$ Engl J Med 2003;349:535-45.

34 Alexandersen P, Haarbo J, Sandholdt I, Shalmi M, Lawaetz H, Christiansen C. Norethindrone acetate enhances the antiatherogenic effect of 17 beta-estradiol : a secondary prevention study of aortic atherosclerosis in ovariectomized cholesterol-fed rabbits. Arterioscler Thromb Vasc Biol 1998;18:902-7.

35 LaCroix AZ, Chlebowski RT, Manson JE, Aragaki AK, Johnson KC, Martin L, et al. Health outcomes after stopping conjugated equine estrogens among postmenopausal women with prior hysterectomy. JAMA 2011;305:1305-14.

36 Prentice RL, Chlebowski RT, Stefanick ML, Manson JE, Pettinger M, Hendrix SL, et al. Estrogen plus progestin therapy and breast cancer in recently postmenopausal women. Am J Epidemiol 2008;167:1207-16.

37 Fournier A, Mesrine S, Boutron-Ruault MC, Clavel-Chapelon F. Estrogen-progestagen menopausal hormone therapy and breast cancer: does delay from menopause onset to treatment initiation influence risks? J Clin Oncol 2009;27:5138-43.

38 Harman SM, Brinton EA, Cedars M, Lobo R, Manson JE, Merriam GR, et al. KEEPS: the Kronos Early Estrogen Prevention Study. Climacteric 2005;8:3-12.

39 Avenell A, Grant AM, McGee M, McPherson G, Campbell MK, McGee MA. The effects of an open design on trial participant recruitment, compliance and retention-a randomized controlled trial comparison with a blinded, placebo-controlled design. Clin Trials 2004;1:490-8.

Accepted: 16 September 2012

\section{Cite this as: BMJ 2012;345:e6409}

This is an open-access article distributed under the terms of the Creative Commons Attribution Non-commercial License, which permits use, distribution, and reproduction in any medium, provided the original work is properly cited, the use is non commercial and is otherwise in compliance with the license. See: http://creativecommons.org/licenses/by$\mathrm{nc} / 2.0 /$ and http://creativecommons.org/licenses/by-nc/2.0/legalcode. 


\section{What is already known on this topic}

Observational studies consistently indicate that hormone replacement therapy (HRT) reduces the risk of coronary heart disease (CHD) Randomised trials show a null effect although most women in these trials were older than 60 and more than 10 years postmenopausal Randomised trials of HRT and meta-analyses indicate that $\mathrm{CHD}$ and total mortality are reduced when HRT is initiated in women aged less than 60 or within 10 years of menopause

\section{What this study adds}

HRT started early in postmenopausal women significantly reduces the risk of the combined endpoint of mortality, myocardial infarction, or heart failure

Postmenopausal women who started HRT early and used it for more than 10 years were not at significantly increased risk of breast cancer or stroke 


\section{Table}

Table 1| Baseline characteristics of participants. Values are means (standard deviations) unless stated otherwise

Characteristics

Age (years)

Body mass index $\left(\mathrm{kg} / \mathrm{m}^{2}\right)$

Hip:waist ratio

Total cholesterol concentration (mmol/L)

Low density lipoprotein concentration ( $\mathrm{mmol} / \mathrm{L}$ )

High density lipoprotein concentration (mmol/L)

Triglyceride concentration ( $\mathrm{mmol} / \mathrm{L}$ )

Systolic blood pressure $(\mathrm{mm} \mathrm{Hg})$

Diastolic blood pressure $(\mathrm{mm} \mathrm{Hg})$

Time since menopause (years)

Fasting glucose concentration ( $\mathrm{mmol} / \mathrm{L}$ )

No (\%) with vitamin $\mathrm{D}$ deficiency

No (\%) of smokers

*Age difference, $\mathrm{P}=0.007$.

Control group $(n=504)$ Hormone replacement therapy group $(n=502)$

$50.0(2.8) \quad 49.5(2.7)^{*}$

$25.2(4.5) \quad 25.3(4.3)$

$1.26(0.12) \quad 1.27(0.11)$

$6.32(0.98) \quad 6.28(1.10)$

$3.82(1.01) \quad 3.85(1.04)$

$1.74(0.48) \quad 1.71(0.42)$

$1.20(0.70) \quad 1.14(0.53)$

$130(20) \quad 129(18)$

$81(11) \quad 81(11)$

$0.61(0.65) \quad 0.58(0.63)$

$4.7(0.7) \quad 4.7(0.7)$

$170(33.7) \quad 169(33.6)$

$225(44.6) \quad 212(42.3)$




\section{Figures}

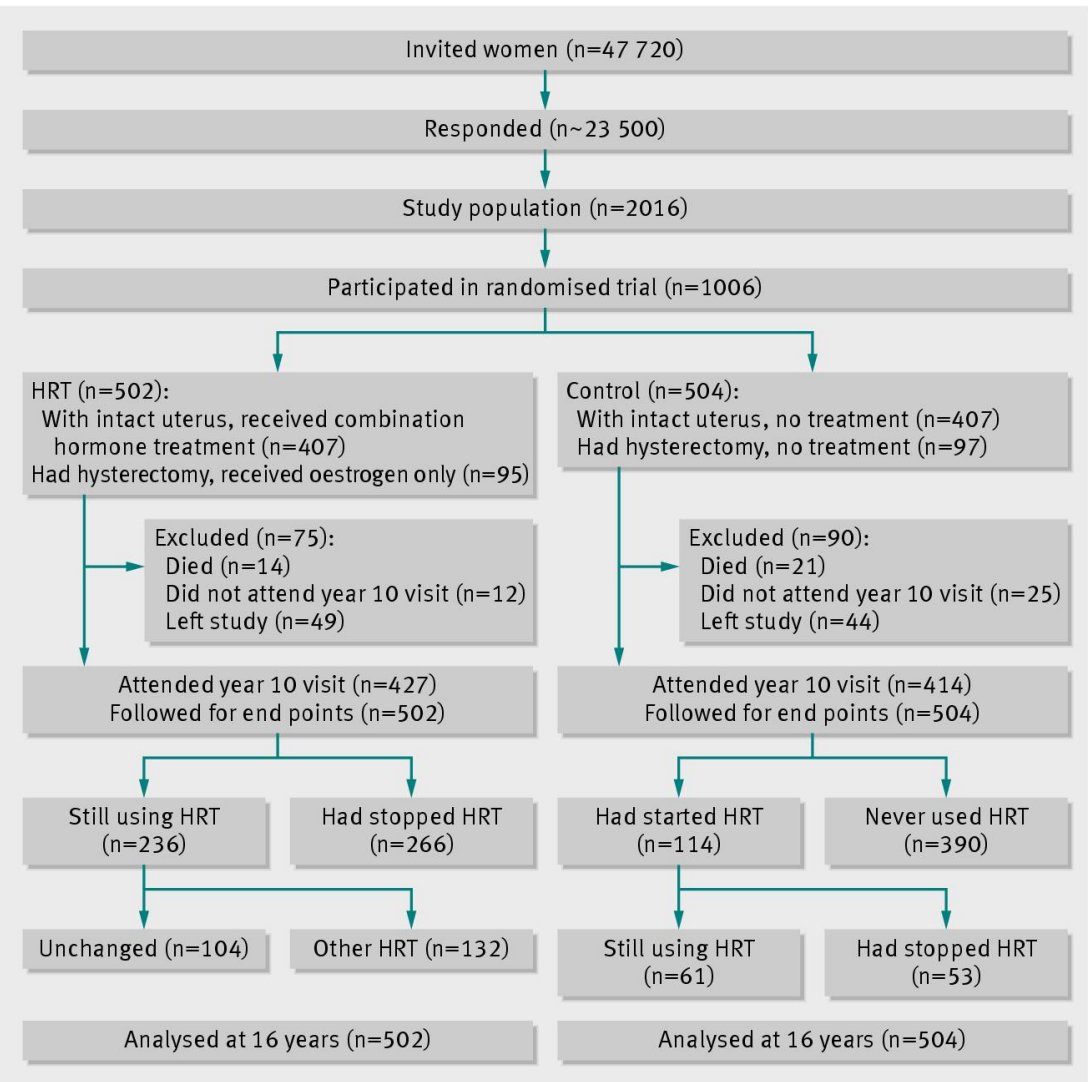

Fig 1 Flow of participants through study. HRT=hormone replacement therapy

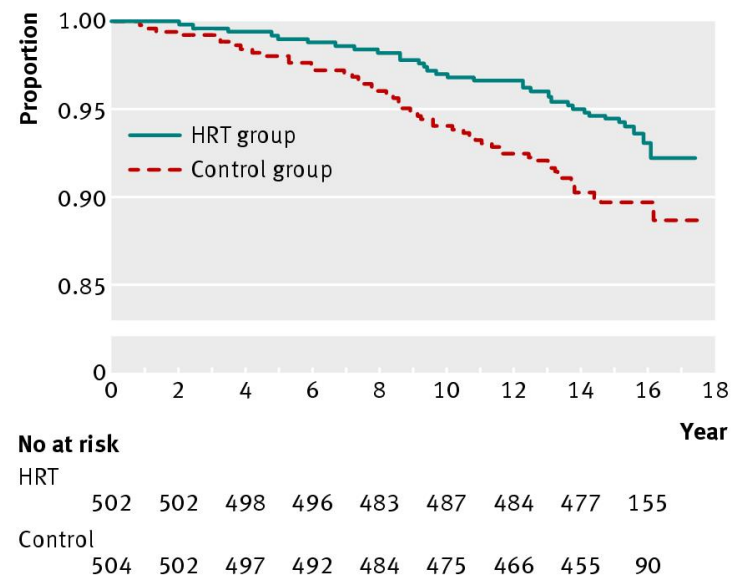

Fig 2 Risk of death or admission to hospital due to heart failure or myocardial infarction (primary endpoint) over 16 years of follow-up including 11 years of randomised treatment 


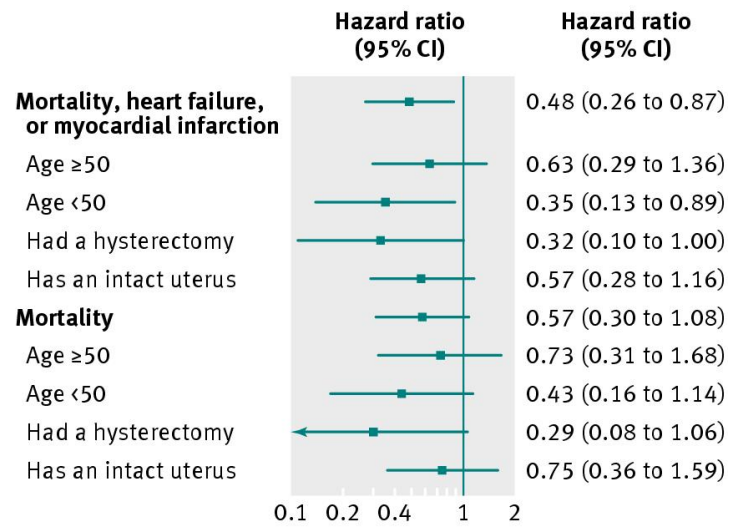

Fig 3 Primary endpoint and mortality for hormone replacement therapy in the total population as well as in four specified subsets of participants during randomisation phase (up to year 2002). Women in the hormone replacement therapy group who had undergone hysterectomy received oestrogen only, whereas women with an intact uterus received combination therapy

\begin{tabular}{|c|c|c|c|}
\hline \multirow[b]{2}{*}{ Deep vein thrombsis } & \multicolumn{2}{|c|}{$\begin{array}{c}\text { Hazard ratio } \\
(95 \% \mathrm{Cl})\end{array}$} & \multirow{2}{*}{$\begin{array}{c}\begin{array}{c}\text { Hazard ratio } \\
(95 \% \mathrm{Cl})\end{array} \\
2.01(0.18 \text { to } 22.16)\end{array}$} \\
\hline & & $\longrightarrow$ & \\
\hline Stroke & & & $0.77(0.35$ to 1.70$)$ \\
\hline Breast cancer & & & $0.58(0.27$ to 1.27$)$ \\
\hline Age $\geq 50$ & & & 0.98 (0.33 to 2.92$)$ \\
\hline Age $<50$ & & & $0.34(0.11$ to 1.08$)$ \\
\hline Had a hysterectomy & & & $0.55(0.16$ to 1.88$)$ \\
\hline Has an intact uterus & & & $0.60(0.21$ to 1.65$)$ \\
\hline Mortality or breast cancer & $\rightarrow$ & & $0.54(0.32$ to 0.91$)$ \\
\hline Age $\geq 50$ & & & 0.77 (0.38 to 1.57$)$ \\
\hline Age $<50$ & 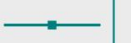 & & 0.36 (0.17 to 0.79$)$ \\
\hline Had a hysterectomy & 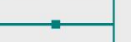 & & 0.38 (0.15 to 0.99$)$ \\
\hline Has an intact uterus & & & 0.64 (0.34 to 1.91$)$ \\
\hline Other cancer & & - & 1.04 (0.60 to 1.80$)$ \\
\hline Age $\geq 50$ & & & 1.34 (0.64 to 2.82$)$ \\
\hline Age $<50$ & $\Rightarrow$ & - & $0.79(0.35$ to 1.79$)$ \\
\hline & 0.5 & & \\
\hline
\end{tabular}

Fig 4 Risk associated with hormone replacement therapy for the different endpoints in total population as well as in four specified subsets during randomisation phase (up to year 2002). Women in the treated group who had undergone hysterectomy received estrogen only, whereas women with an intact uterus received combination therapy

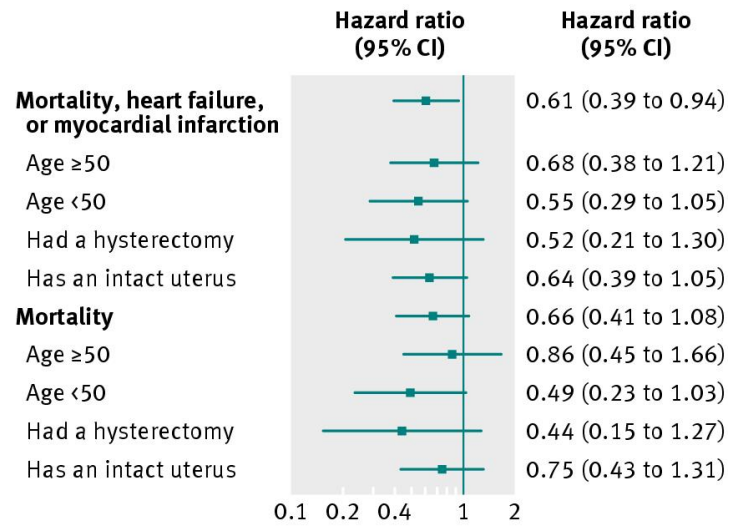

Fig 5 Primary endpoint and mortality for hormone replacement therapy in total population and in four specified subsets of participants, 16 years data including 11 years of randomised treatment. Women in the treated group who had undergone hysterectomy received oestrogen only, whereas women with an intact uterus received combination therapy 


\begin{tabular}{|c|c|c|}
\hline & $\begin{array}{c}\text { Hazard ratio } \\
(95 \% \mathrm{Cl})\end{array}$ & $\begin{array}{c}\text { Hazard ratio } \\
(95 \% \mathrm{Cl})\end{array}$ \\
\hline Deep vein thrombsis & & 0.80 (0.22 to 2.99) \\
\hline Pulmonary embolism & & 0.33 (0.04 to 3.21) \\
\hline Stroke & & 0.89 (0.48 to 1.65$)$ \\
\hline Breast cancer & & $0.90(0.52$ to 1.57$)$ \\
\hline Age $\geq 50$ & & 1.58 (0.73 to 3.44$)$ \\
\hline Age $<50$ & & $0.50(0.22$ to 1.14$)$ \\
\hline Had a hysterectomy & & 0.63 (0.23 to 1.78$)$ \\
\hline Has an intact uterus & & 1.05 (0.54 to 2.04$)$ \\
\hline Mortality or breast cancer & & 0.73 (0.50 to 1.06$)$ \\
\hline Age $\geq 50$ & - & $1.04(0.62$ to 1.74$)$ \\
\hline Age $<50$ & & $0.49(0.28$ to 0.87$)$ \\
\hline Had a hysterectomy & & 0.42 (0.18 to 0.97$)$ \\
\hline Has an intact uterus & & $0.86(0.55$ to 1.32$)$ \\
\hline Other cancer & - & 1.21 (0.81 to 1.82$)$ \\
\hline Age $\geq 50$ & $=$ & 1.18 (0.68 to 2.05$)$ \\
\hline Age $<50$ & $=-$ & 1.28 (0.70 to 2.32$)$ \\
\hline & 0.51234 & \\
\hline
\end{tabular}

Fig 6 Risk associated with hormone replacement therapy for different endpoints in total population as well as in four specified subsets of participants, 16 years data including 11 years of randomised treatment. Women in the treated group with hysterectomy received oestrogen only, whereas women with intact uterus received combination therapy 